\title{
Reflections at infinity of time changed RBMs on a domain with Liouville branches
}

\author{
By Zhen-Qing Chen and Masatoshi Fukushima
}

(Received Oct. 25, 2016)

\begin{abstract}
Let $Z$ be the transient reflecting Brownian motion on the closure of an unbounded domain $D \subset \mathbb{R}^{d}$ with $N$ number of Liouville branches. We consider a diffuion $X$ on $\bar{D}$ having finite lifetime obtained from $Z$ by a time change. We show that $X$ admits only a finite number of possible symmetric conservative diffusion extensions $Y$ beyond its lifetime characterized by possible partitions of the collection of $N$ ends and we identify the family of the extended Dirichlet spaces of all $Y$ (which are independent of time change used) as subspaces of the space BL $(D)$ spanned by the extended Sobolev space $H_{e}^{1}(D)$ and the approaching probabilities of $Z$ to the ends of Liouville branches.
\end{abstract}

\section{Introduction.}

The boundary problem of a Markov process $X$ concerns all possible Markovian prolongations $Y$ of $X$ beyond its life time $\zeta$ whenever $\zeta$ is finite. For a conservative but transient Markov process, we can still consider its extension, after a time change to speed up the original process. Let $Z=\left(Z_{t}, \mathbf{Q}_{x}\right)$ be a conservative right process on a locally compact separable metric space $E$ and $\partial$ be the point at infinity of $E$. Suppose $Z$ is transient relative to an excessive measure $m$ : for the 0 -order resolvent $R$ of $Z, R f(z)<\infty, m$-a.e. for some strictly positive function (or equivalently, for any non-negative function) $f \in L^{1}(E ; m)$. Then

$$
\mathbf{Q}_{x}\left(\lim _{t \rightarrow \infty} Z_{t}=\partial\right)=1 \quad \text { for q.e. } x \in E,
$$

if $R f$ is lower semicontinuous for any non-negative Borel function $f$ ([FTa $]$ ). The last condition is not needed when $X$ is $m$-symmetric ([CF2]). Here, 'q.e.' means 'except for an $m$-polar set'.

Take any strictly positive bounded function $f \in L^{1}(E ; m)$. Then $A_{t}=\int_{0}^{t} f\left(Z_{s}\right) d s$, $t \geq 0$ is a strictly increasing PCAF of $Z$ with $\mathbf{E}_{x}^{\mathbf{Q}}\left[A_{\infty}\right]=R f(x)<\infty$ for q.e. $x \in E$. The time changed process $X=\left(X_{t}, \zeta, \mathbf{P}_{x}\right)$ of $Z$ by means of $A$ is defined by

$$
X_{t}=Z_{\tau_{t}}, t \geq 0, \quad \tau=A^{-1}, \quad \zeta=A_{\infty}, \quad \mathbf{P}_{x}=\mathbf{Q}_{x}, x \in E .
$$

Since $\mathbf{P}_{x}\left(\zeta<\infty, \lim _{t \rightarrow \zeta} X_{t}=\partial\right)=\mathbf{P}_{x}(\zeta<\infty)=1$ for q.e. $x \in E$, the boundary problem for $X$ at $\partial$ makes perfect sense. We denote $X$ also by $X^{f}$ to indicate its dependence on the function $f$. For different choices of $f, X^{f}$ have a common geometric structure related

2010 Mathematics Subject Classification. Primary 60J50; Secondary 60J65, 31C25.

Key Words and Phrases. transient reflecting Brownian motion, time change, Liouville domain, Beppo Levi space, approaching probability, quasi-homeomorphism, zero flux. 
each other only by time changes. Making a closer look at the geometric behaviors of a conservative transient process $Z$ around $\partial$ is a right way toward the study of the boundary problem for $X=X^{f}$. A strong Markov process $\widehat{X}$ on a topological space $\widehat{E}$ is said to be an extension of $X$ on $E$ if (i) $E$ can be embedded homomorphically as a dense open subset of $\widehat{E}$, (ii) the part process of $\widehat{X}$ killed upon leaving $E$ has the same distribution as $X$, and (iii) $\widehat{X}$ has no sojourn on $\widehat{E} \backslash E$; that is, $\widehat{X}$ spends zero Lebesgue amount of time on $\widehat{E} \backslash E$.

In this paper, $Z$ is the transient reflecting Brownian motion on the closure of an unbounded domain $D \subset \mathbb{R}^{d}$ with $N$ number of Liouville branches. Our main aim is to prove in Section 5 that a time changed process $X^{f}$ of $Z$ admits essentially only a finite number of possible symmetric conservative diffusion extensions $Y$ beyond its lifetime. They are characterized by the partition of the collection of $N$ ends. Moreover, all the corresponding extended Dirichlet spaces $\left(\mathcal{E}^{Y}, \mathcal{F}_{e}^{Y}\right)$ are identified in terms of the extended Dirichlet space of $Z$ and the approaching probabilities of $Z$ to the ends of Liouville branches in an extremely simple manner. These extended Dirichlet spaces are independent of the choice of $f$. The $L^{2}$-generator of each extension $Y$ is also characterized in Section 6 by means of zero flux conditions at the ends of branches. Each extension $Y$ may be called a many point reflection at infinity of $X^{f}$ generalizing the notion of the one point reflection in [CF3] in the present specific context. The characterization of possible extensions also uses quasi-homeomorphism and equivalence between Dirichlet forms. See the Appendix, Section 8, of this paper for details.

In fact, our results are valid for a time changed process $X^{\mu}$ of $Z$ by means of a more general finite smooth measure $\mu$ on $\bar{D}$ than $f(x) d x$. This is demonstrated in Section 7 .

Although we formulate our results for the reflecting Brownian motion on an unbounded domain in $\mathbb{R}^{d}$ with several Liouville branches, all of them except for Theorem 6.1 remain valid without any essential change for the reflecting diffusion process associated with the uniformly elliptic second order self-adjoint partial differential operator with measurable coefficients that was constructed in $[\mathbf{C}]$ and $[$ FTo]. Since we need strong Feller property of the reflecting diffusion process, we assume the underlying unbounded domain is Lipschitz in the sense of [FTo]; see Remark 5.3. Thus we are effectively investigating common path behaviors at infinity holding for such a general family of diffusion processes.

ACKnowledgements. This paper is a direct outgrowth of our paper $[\mathbf{C F 1}]$ and Chapter 7 of our book [CF2]. In relation to them, we had very valuable discussions with Krztsztof Burdzy on boundaries of transient reflecting Brownian motions. We would like to express our sincere thanks to him.

\section{Preliminaries.}

For a domain $D \subset \mathbb{R}^{d}$, let us consider the spaces

$$
\mathrm{BL}(D)=\left\{u \in L_{\mathrm{loc}}^{2}(D):|\nabla u| \in L^{2}(D)\right\}, \quad H^{1}(D)=\mathrm{BL}(D) \cap L^{2}(D) .
$$

The space $\mathrm{BL}(D)$ called the Beppo Levi space was introduced by Deny and Lions [DL] as the space of Schwartz distributions whose first order derivatives are in $L^{2}(D)$, which 
can be identified with the function space described above. The quotient space BL $(D)$ of $\mathrm{BL}(D)$ by the space of all constant functions on $D$ is a real Hilbert space with inner product

$$
\mathbf{D}(u, v)=\int_{D} \nabla u(x) \cdot \nabla v(x) d x .
$$

See Section 1.1 of Maz'ja $[\mathbf{M}]$ for proofs of the above stated facts, where the space $\mathrm{BL}(D)$ is denoted by $L_{2}^{1}(D)$ and studied in a more general context of the spaces $L_{p}^{\ell}(D), \ell \geq$ $1, p \geq 1$.

Define

$$
(\mathcal{E}, \mathcal{F})=\left(\frac{1}{2} \mathbf{D}, H^{1}(D)\right),
$$

which is a Dirichlet form on $L^{2}(D)$. The collection of those domains $D \subset \mathbb{R}^{d}$ for which (2.2) is regular on $L^{2}(\bar{D})$ will be denoted by $\mathcal{D}$. It is known that $D \in \mathcal{D}$ if $D$ is either a domain of continuous boundary or an extendable domain relative to $H^{1}(D)$ (cf. [CF1, p.866]). For $D \in \mathcal{D}$, the diffusion process $Z$ on $\bar{D}$ associated with (2.2) is by definition the reflecting Brownian motion (RBM in abbreviation) which is known to be conservative. Furthermore, the space $\mathrm{BL}(D)$ is nothing but the reflected Dirichlet space of the form (2.2) ([CF2, Section 6.5]). The Dirichlet form (2.2) is either recurrent or transient and the latter case occurs only when $d \geq 3$ and $D$ is unbounded. For $D_{1}, D_{2} \in \mathcal{D}$ with $D_{1} \subset D_{2},(2.2)$ is transient for $D_{2}$ whenever so it is for the smaller domain $D_{1}$. If $(2.2)$ is recurrent, then we have the identity

$$
\mathrm{BL}(D)=H_{e}^{1}(D)
$$

where $H_{e}^{1}(D)$ denotes the extended Dirichlet space of the form (2.2) or of the RBM $Z$ ([CF2] $)$ that may be called the extended Sobolev space of order 1.

Suppose $D \in \mathcal{D}$ and $(2.2)$ is transient. Then $H_{e}^{1}(D)$ is a Hilbert space with inner product $\mathbf{D} / 2$ possessing the space $C_{c}^{\infty}(\bar{D})$ as its core. $H_{e}^{1}(D)$ can be regarded as a proper closed subspace of the quotient space BL $(D)$. Define

$$
\mathcal{H}^{*}(D)=\left\{u \in \operatorname{BL}(D): \mathbf{D}(u, v)=0 \text { for every } v \in H_{e}^{1}(D)\right\} .
$$

Any function $u \in \mathrm{BL}(D)$ admits a unique decomposition

$$
u=u_{0}+h, \quad u_{0} \in H_{e}^{1}(D), \quad h \in \mathcal{H}^{*}(D) .
$$

Any function $h \in \mathcal{H}^{*}(D)$ is of finite Dirichlet integral and harmonic on $D$. Furthermore, the quasi-continuous version of $h$ is harmonic on $\bar{D}$ with respect to the RBM $Z$.

In what follows, we restrict our attention to the case where the form (2.2) is transient and so we assume that $d \geq 3$ and $D \in \mathcal{D}$ is unbounded.

Definition 2.1. A domain $D \in \mathcal{D}$ is called a Liouville domain if the Dirichlet form $(2.2)$ is transient and $\operatorname{dim} \mathcal{H}^{*}(D)=1$. 
A domain $D \in \mathcal{D}$ is a Liouville domain if and only if the form (2.2) is transient and any function $u \in \operatorname{BL}(D)$ admits a unique decomposition

$$
u=u_{0}+c, \quad \text { where } u_{0} \in H_{e}^{1}(D) \text { and } c \in \mathbb{R} .
$$

We shall denote by $c(u)$ the constant $c$ in (2.5) uniquely associated with $u \in \operatorname{BL}(D)$ for a Liouville domain $D$.

A trivial but important example of a Liouville domain is $\mathbb{R}^{d}$ with $d \geq 3$, see Brelot [B]. Another important example of a Liouville domain is provided by an unbounded uniform domain that has been shown by Jones $[\mathbf{J}]$ (see also $[\mathbf{H K}]$ ) to be an extendable domain relative to the space $\mathrm{BL}(D)$.

A domain $D \subset \mathbb{R}^{d}$ is called a uniform domain if there exists $C>0$ such that for every $x, y \in D$, there is a rectifiable curve $\gamma$ in $D$ connecting $x$ and $y$ with length $(\gamma) \leq C|x-y|$, and moreover

$$
\min \{|x-z|,|z-y|\} \leq C \operatorname{dist}\left(z, D^{c}\right) \quad \text { for every } z \in \gamma .
$$

It was proved in Theorem 3.5 of [CF1] that any unbounded uniform domain is a Liouville domain in the sense of Definition 2.1. An unbounded uniform domain is such a domain that is broaden toward the infinity. The truncated infinite cone $C_{A, a}=\{(r, \omega): r>a$, $\omega \in A\} \subset \mathbb{R}^{d}$ for any connected open set $A \subset S^{d-1}$ with Lipschitz boundary is an unbounded uniform domain. To the contrary, (2.2) is recurrent for the cylinder $D=$ $\left\{\left(x, x^{\prime}\right) \in \mathbb{R}^{d}: x \in \mathbb{R},\left|x^{\prime}\right|<1\right\}$. See Pinsky $[\mathbf{P}]$ for transience criteria for other types of domains. On the other hand, it has been shown in [CF2, Proposition 7.8.5] that (2.2) is transient but $\operatorname{dim}\left(\mathcal{H}^{*}(D)\right)=2$ for a special domain

$$
D=B_{1}(\mathbf{0}) \cup\left\{\left(x, x^{\prime}\right) \in \mathbb{R}^{d}: x \in \mathbb{R},|x|>\left|x^{\prime}\right|\right\}, \quad d \geq 3
$$

with two symmetric cone branches. Here $B_{r}(\mathbf{0}), r>0$, denotes an open ball with radius $r$ centered at the origin. This domain is not uniform because of a presence of a bottleneck. We shall consider much more general domains than this. But before proceeding to the main setting of the present paper, we state a simple property of Liouville domains:

Proposition 2.2. For $D_{1}, D_{2} \in \mathcal{D}$ with $D_{1} \subset D_{2}$, suppose $D_{1}$ is a Liouville domain and $D_{2} \backslash D_{1}$ is bounded. Then $D_{2}$ is a Liouville domain. Furthermore, for any $u \in \operatorname{BL}\left(D_{2}\right)$, it holds that $c(u)=c\left(\left.u\right|_{D_{1}}\right)$.

Proof. The proof is similar to that of [CF1, Proposition 3.6]. Note that (2.2) is transient for $D_{2}$. We show that any $u \in \mathrm{BL}\left(D_{2}\right)$ admits a decomposition (2.5) with $u_{0} \in H_{e}^{1}\left(D_{2}\right)$ and $c=c\left(\left.u\right|_{D_{1}}\right)$. Due to the normal contraction property of $\operatorname{BL}\left(D_{2}\right)$ and the transience of $\left(\mathbf{D} / 2, H^{1}(D)\right)$, we may assume that $u$ is bounded on $D_{2}$. By noting that $\left.u\right|_{D_{1}} \in \mathrm{BL}\left(D_{1}\right)$ and $D_{1}$ is a Liouville domain, we let $c=c\left(\left.u\right|_{D_{1}}\right)$ and $u_{0}(x)=u(x)-c, x \in D_{2}$. Then $\left.u_{0}\right|_{D_{1}} \in H_{e}^{1}\left(D_{1}\right)$. To prove that $u_{0} \in H_{e}^{1}\left(D_{2}\right)$, choose an open ball $B_{r}(\mathbf{0}) \supset \overline{D_{2} \backslash D_{1}}$ and a function $w \in C_{c}^{\infty}\left(\mathbb{R}^{d}\right)$ with $w(x)=1, x \in B_{r}(\mathbf{0})$. Clearly $w u_{0} \in H_{e}^{1}\left(D_{2}\right)$.

It remains to show $(1-w) u_{0} \in H_{e}^{1}\left(D_{2}\right)$. Take $g_{n} \in H^{1}\left(D_{1}\right)$ converging to $u_{0}$ a.e. on 
$D_{1}$ and in the Dirichlet norm on $D_{1}$. By truncation, we may assume that $g_{n}$ is uniformly bounded on $D_{1}$. Then

$$
\begin{aligned}
& \int_{D_{2}}\left|\nabla\left[(1-w(x)) g_{n}(x)\right]\right|^{2} d x \\
\leq & 2 \sup _{x \in \mathbb{R}^{d}}(1-w(x))^{2} \int_{D_{1}}\left|\nabla g_{n}(x)\right|^{2} d x+2 \sup _{x \in D_{1}}\left|g_{n}(x)\right|^{2} \int_{\mathbb{R}^{d}}|\nabla w(x)|^{2} d x,
\end{aligned}
$$

which is uniformly bounded in $n$, yielding by the Banach-Saks theorem that $(1-w) u_{0} \in$ $H_{e}^{1}\left(D_{2}\right)$.

We shall work under the regularity condition

(A.1) $D$ is of a Lipschitz boundary $\partial D$,

which means the following: there are constants $M>0, \delta>0$ and a locally finite covering $\left\{U_{j}\right\}_{j \in J}$ of $\partial D$ such that, for each $j \in J, D \cap U_{j}$ is a upper part of a graph of a Lipschitz continuous function under an appropriate coodinate system with the Lipschitz constant bounded by $M$ and $\partial D \subset \bigcup_{j \in J}\left\{x \in U_{j}: \operatorname{dist}\left(x, \partial U_{j}\right)>\delta\right\}$. According to [FTo], there exists then a conservative diffusion process $Z=\left(Z_{t}, \mathbf{Q}_{x}\right)$ on $\bar{D}$ associated with the regular Dirichlet form $(2.2)$ on $L^{2}(\bar{D})$ whose resolvent $\left\{G_{\alpha}^{Z} ; \alpha>0\right\}$ has the strong Feller property in the sense that

$$
G_{\alpha}^{Z}\left(b L^{1}(D)\right) \subset b C(\bar{D}) .
$$

$Z$ is a precise version of the RBM on $\bar{D}$. In particular, the transition probability of $Z$ is absolutely continuous with respect to the Lebesgue measure.

Under the condition (A.1) and the transience assumption on (2.2), the RBM $Z=$ $\left(Z_{t}, \mathbf{Q}_{x}\right)$ on $\bar{D}$ enjoys the properties that

$$
\mathbf{Q}_{x}\left(\lim _{t \rightarrow \infty} Z_{t}=\partial\right)=1 \quad \text { for every } x \in \bar{D}
$$

where $\partial$ denotes the point at infinity of $\bar{D}$, and

$$
\mathbf{Q}_{x}\left(\lim _{t \rightarrow \infty} u\left(Z_{t}\right)=0\right)=1 \quad \text { for every } x \in \bar{D},
$$

for any $u \in H_{e}^{1}(D), u$ being taken to be quasi-continuous. See [CF2, Section 7.8, $\left.\left(4^{o}\right)\right]$.

In the rest of this paper, we fix a domain $D$ of $\mathbb{R}^{d}, d \geq 3$, satisfying (A.1) and

$$
D \backslash \overline{B_{r}(\mathbf{0})}=\bigcup_{j=1}^{N} C_{j}
$$

for some $r>0$ and an integer $N$, where $C_{1}, \ldots, C_{N}$ are Liouville domains with Lipschitz boundaries such that $\bar{C}_{1}, \ldots, \bar{C}_{N}$ are mutually disjoint. $D$ may be called a Lipschitz domain with $N$ number of Liouville branches.

Let $\partial_{j}$ be the point at infinity of the unbounded closed set $\bar{C}_{j}$ for each $1 \leq j \leq N$. Denote the $N$-points set $\left\{\partial_{1}, \ldots, \partial_{N}\right\}$ by $F$ and put $\bar{D}^{*}=\bar{D} \cup F . \bar{D}^{*}$ can be made to be a compact Hausdorff space if we employ as a local base of neighborhoods of each 
point $\partial_{j} \in F$ the neighborhoods of $\partial_{j}$ in $\bar{C}_{j} \cup\left\{\partial_{j}\right\} . \bar{D}^{*}$ may be called the $N$-points compactification of $\bar{D}$.

Obviously the Dirichlet form (2.2) is transient for $D$. We shall verify in Section 4 that $\operatorname{dim}\left(\mathcal{H}^{*}(D)\right)=N$. Here we note the following implication of Proposition 2.2; if a domain $D$ is of the type (A.2) for different $0<r_{1}<r_{2}$, and if $D$ is a domain with $N$ number of Liouville branches relative to $r_{2}$, then so it is relative to $r_{1}$.

\section{Approaching probabilities of $\mathrm{RBM} Z$ and limits of BL-functions along $Z_{t}$.}

For each $1 \leq j \leq N$, define the approaching probability of the $\operatorname{RBM} Z=\left(Z_{t}, \mathbf{Q}_{x}\right)$ to $\partial_{j}$ by

$$
\varphi_{j}(x)=\mathbf{Q}_{x}\left(\lim _{t \rightarrow \infty} Z_{t}=\partial_{j}\right), \quad x \in \bar{D} .
$$

Proposition 3.1. It holds that

$$
\sum_{j=1}^{N} \varphi_{j}(x)=1 \quad \text { for every } \quad x \in \bar{D},
$$

and, for each $1 \leq j \leq N$,

$$
\varphi_{j}(x)>0 \quad \text { for every } \quad x \in \bar{D} \text {. }
$$

Proof. (3.2) is a consequence of (2.8). As $\varphi_{j}$ is a non-negative harmonic function on the domain $D$, it is either identically zero on $D$ or strictly positive on $D$. Since $\varphi_{j}(x)=Q_{t} \varphi_{j}(x), x \in \bar{D}$, where $Q_{t}$ is the transition semigroup of the RBM $Z$, which has a strictly positive transition density kernel, the above dichotomy extends from $D$ to $\bar{D}$.

Suppose $\varphi_{j}(x) \equiv 0$ on $\bar{D}$. Then by $(2.8)$

$$
\mathbf{Q}_{x}\left(\sigma_{\partial B_{r}(\mathbf{0})}<\infty\right)=1, \quad \text { for any } x \in \bar{C}_{j} \backslash B_{r+1}(\mathbf{0}) .
$$

Let $Z^{j}=\left(Z_{t}^{j}, \mathbf{Q}_{x}^{j}\right), x \in \bar{C}_{j}$, be the $\mathrm{RBM}$ on $\bar{C}_{j}$, which is transient as $C_{j}$ is a Liouville domain. Since $Z$ and $Z^{j}$ share the common part process on $\bar{C}_{j} \backslash \partial B_{r}(\mathbf{0})$, (3.4) remains valid if $\mathbf{Q}_{x}$ is replaced by $\mathbf{Q}_{x}^{j}$. By the Markov property of $Z^{j}$ and the conservativeness of $Z^{j}$, we have

$$
\mathbf{Q}_{x}^{j}\left(\sigma_{\partial B_{r}(\mathbf{0})} \circ \theta_{\ell}<\infty \text { for every integer } \ell\right)=1,
$$

for any $x \in \bar{C}_{j} \backslash B_{r+1}(\mathbf{0})$. This however contradicts to the transience property (2.8) of $Z^{j}$.

Proposition 3.2. For any $u \in \operatorname{BL}(D)$, let $c_{j}(u)=c\left(\left.u\right|_{C_{j}}\right)$ for $1 \leq j \leq N$. Then

$$
\mathbf{Q}_{x}\left(Z_{\infty-}=\partial_{j}, \lim _{t \rightarrow \infty} u\left(Z_{t}\right)=c_{j}(u)\right)=\mathbf{Q}_{x}\left(Z_{\infty-}=\partial_{j}\right), x \in \bar{D}, \quad 1 \leq j \leq N .
$$

If $c_{j}(u)=0$ for every $1 \leq j \leq N$, then $u \in H_{e}^{1}(D)$. 
Proof. We prove (3.5) for $j=1$. Let $r>0$ be the radius in (A.2) and $Z^{1}=$ $\left(Z_{t}^{1}, \mathbf{Q}_{x}^{1}\right)$ be the RBM on $\overline{C_{1}}$. The hitting times of $B_{r}(\mathbf{0})$ and $B_{R}(\mathbf{0})$ for $R>r$ will be denoted by $\sigma_{r}$ and $\sigma_{R}$, respectively. Observe that $Z$ and $Z^{1}$ share in common the part process on $\bar{C}_{1} \backslash \partial B_{r}(\mathbf{0})$. Since $C_{1}$ is a Liouville domain, we see from (2.5) and (2.9) that

$$
\mathbf{Q}_{x}^{1}\left(\lim _{t \rightarrow \infty} u\left(Z_{t}^{1}\right)=c_{1}(u)\right)=1 \quad \text { for every } x \in \bar{C}_{1} .
$$

For $R>r$, we consider the event

$$
\Gamma_{R}=\left\{Z_{\sigma_{R}} \in \bar{C}_{1}, \sigma_{r} \circ \theta_{\sigma_{R}}=\infty\right\} .
$$

Then $\Gamma_{R} \cap\left\{Z_{\infty_{-}}=\partial\right\}$ increases as $R$ increases and $\left\{Z_{\infty_{-}}=\partial_{1}\right\}=\bigcup_{R>r}\left[\Gamma_{R} \cap\left\{Z_{\infty_{-}}=\right.\right.$ $\partial\}$. In view of (2.8), we have for $x \in \bar{D}$,

$$
\begin{aligned}
\mathbf{Q}_{x}\left(Z_{\infty-}=\partial_{1}\right) & =\lim _{R \rightarrow \infty} \mathbf{Q}_{x}\left(\Gamma_{R} \cap\left\{Z_{\infty-}=\partial\right\}\right)=\lim _{R \rightarrow \infty} \mathbf{Q}_{x}\left(\Gamma_{R}\right) \\
& =\lim _{R \rightarrow \infty} \mathbf{E}^{\mathbf{Q}_{x}}\left[\mathbf{Q}_{Z_{\sigma_{R}}}\left(\sigma_{r}=\infty\right) ; Z_{\sigma_{R}} \in \bar{C}_{1}\right] \\
& =\lim _{R \rightarrow \infty} \mathbf{E}^{\mathbf{Q}_{x}}\left[\mathbf{Q}_{Z_{\sigma_{R}}}^{1}\left(\sigma_{r}=\infty\right) ; Z_{\sigma_{R}} \in \bar{C}_{1}\right] \\
& =\lim _{R \rightarrow \infty} \mathbf{E}^{\mathbf{Q}_{x}}\left[\mathbf{Q}_{Z_{\sigma_{R}}}^{1}\left(\sigma_{r}=\infty, \lim _{t \rightarrow \infty} u\left(Z_{t}^{1}\right)=c_{1}(u)\right) ; Z_{\sigma_{R}} \in \bar{C}_{1}\right] .
\end{aligned}
$$

In exactly the same way, we can see that $\mathbf{Q}_{x}\left(Z_{\infty-}=\partial_{1}, \lim _{t \rightarrow \infty} u\left(Z_{t}\right)=c_{1}(u)\right)$ equals the last expression in the above display, proving (3.5) for $j=1$

Suppose $u \in \operatorname{BL}(D)$ satisfies $c_{j}(u)=0$ for every $1 \leq j \leq N$. Then $\left.u\right|_{C_{j}} \in H_{e}^{1}\left(C_{j}\right)$ for every $1 \leq j \leq N$ and we can conclude as the proof of Proposition 2.2 that $u \in H_{e}^{1}(D)$.

We remark that, in view of Proposition 2.2 the constants $c_{j}(u), 1 \leq j \leq N$, in the above proposition are independent of the choice of the radius $r$ in (A.2).

\section{Reflecting extension $X^{*}$ of a time changed RBM $X$ and dimension of $\mathcal{H}^{*}(D)$.}

Fix a strictly positive bounded integrable function $f$ on $\bar{D}$ and define

$$
A_{t}=\int_{0}^{t} f\left(Z_{s}\right) d s, \quad t \geq 0 .
$$

$A_{t}$ is a positive continuous additive functional (PCAF) of the $\operatorname{RBM} Z=\left(Z_{t}, \mathbf{Q}_{x}\right)$ on $\bar{D}$ in the strict sense with full support. Notice that

$$
\mathbf{Q}_{x}\left(A_{\infty}<\infty\right)=1 \text { for every } x \in \bar{D},
$$

because $\mathbf{E}^{Q_{x}}\left[A_{\infty}\right]=G_{0+}^{Z} f(x)<\infty$ for a.e. $x \in \bar{D}$ due to the transience of $Z$ ([CF2, Proposition 2.1.3]) and hence

$$
\mathbf{Q}_{x}\left(A_{\infty}=\infty\right)=\mathbf{Q}_{x}\left(A_{\infty} \circ \theta_{t}=\infty\right)=\mathbf{E}^{\mathbf{Q}_{x}}\left[\mathbf{Q}_{Z_{t}}\left(A_{\infty}=\infty\right)\right]=0 \quad \text { for every } x \in \bar{D},
$$


on account of the stated absolute continuity of the transition function of $Z$.

Let $X=\left(X_{t}, \zeta, \mathbf{P}_{x}\right)$ be the time changed process of $Z$ by means of $A$ :

$$
X_{t}=Z_{\tau_{t}}, \quad \tau=A^{-1}, \quad \zeta=A_{\infty}, \quad \mathbf{P}_{x}=\mathbf{Q}_{x} \text { for } x \in \bar{D} .
$$

The Markov process $X=X^{f}$ is a diffusion process on $\bar{D}$ symmetric with respect to the measure $m(d x)=f(x) d x$ and the Dirichlet form $\left(\mathcal{E}^{X}, \mathcal{F}^{X}\right)$ of $X$ on $L^{2}(\bar{D} ; m)$ is given by

$$
\mathcal{E}^{X}=\frac{1}{2} \mathbf{D}, \quad \mathcal{F}^{X}=H_{e}^{1}(D) \cap L^{2}(\bar{D} ; m) .
$$

Since the extended Dirichlet space and the reflected Dirichlet space are invariant under a time change by a fully supported PCAF ([CF2, Corollary 5.2.12, Proposition 6.4.6]), these spaces for $\mathcal{E}^{X}$ are still given by $H_{e}^{1}(D)$ and $\mathrm{BL}(D)$, respectively. But the life time $\zeta$ of $X$ is finite $\mathbf{P}_{x}$-a.s. for every $x \in \bar{D}$ in view of (4.2) so that we may consider the problem of extending $X$ after $\zeta$, particularly, from $\bar{D}$ to its $N$-points compactification $\bar{D}^{*}=\bar{D} \cup F$ with $F=\left\{\partial_{1}, \ldots, \partial_{N}\right\}$.

We can rewrite the approaching probability $\varphi_{j}$ of $Z$ to $\partial_{j}$ defined by (3.1) as

$$
\varphi_{j}(x)=\mathbf{P}_{x}\left(\zeta<\infty, \quad X_{\zeta-}=\partial_{j}\right), \quad x \in \bar{D}, \quad 1 \leq j \leq N,
$$

in terms of the time changed process $X$. The measure $m(d x)=f(x) d x$ is extended from $\bar{D}$ to $\bar{D}^{*}$ by setting $m(F)=0$. An $m$-symmetric conservative diffusion process $X^{*}$ on $\bar{D}^{*}$ will be called a symmetric conservative diffusion extension of $X$ if its part process on $\bar{D}$ being killed upon hitting $F$ is equivalent in law with $X$. The resolvent of $X$ is denoted by $\left\{G_{\alpha}^{X}, \alpha>0\right\}$.

Proposition 4.1. $\quad$ There exists a unique symmetric conservative diffusion extension $X^{*}$ of $X$ from $\bar{D}$ to $\bar{D}^{*}=\bar{D} \cup F$. The process $X^{*}$ is recurrent. Let $\left(\mathcal{E}^{*}, \mathcal{F}^{*}\right)$ and $\mathcal{F}_{e}^{*}$ be the Dirichlet form of $X^{*}$ on $L^{2}\left(\bar{D}^{*}, m\right)\left(=L^{2}(D ; m)\right)$ and its extended Dirichlet space, respectively. Then

$$
\begin{gathered}
\mathcal{F}_{e}^{*}=H_{e}^{1}(D) \oplus\left\{\sum_{j=1}^{N} c_{j} \varphi_{j}: c_{j} \in \mathbb{R}\right\} \subset \mathrm{BL}(D), \\
\mathcal{E}^{*}(u, v)=\frac{1}{2} \mathbf{D}(u, v), \quad u, v \in \mathcal{F}_{e}^{*} .
\end{gathered}
$$

Proof. We apply a general existence theorem of a many-point extension formulated in [CF2, Theorem 7.7.4] to the $m$-symmetric diffusion $X$ on $\bar{D}$ and the $N$-points compactification $\bar{D}^{*}=\bar{D} \cup F$ of $\bar{D}$. We verify conditions (M.1), (M.2), (M.3) for $X$ required in this theorem. $\psi_{j}(x):=\mathbf{P}_{x}\left(\zeta<\infty, X_{\zeta-}=\partial_{j}\right)$ is positive for every $x \in \bar{D}, 1 \leq$ $j \leq N$, by (3.3) and (4.5), and so (M.1) is satisfied. Since $m(\bar{D})=\int_{\bar{D}} f d x<\infty$, the $m$-integrability (M.2) of the function $u_{\alpha}^{(j)}(x)=\mathbf{E}_{x}\left[e^{-\alpha \zeta} ; X_{\zeta-}=\partial_{j}\right], x \in \bar{D}$, is trivially fulfilled, $1 \leq j \leq N$. For any $1 \leq j \leq N$ and any compact set $V \subset \bar{D}$, $\inf _{x \in V} G_{\alpha}^{X} \psi_{j}(x)$ is positive because $G_{\alpha}^{X} \psi_{j}=G_{0+}^{X} u_{\alpha}^{(j)}=G_{0+}^{Z}\left(u_{\alpha}^{(j)} f\right)$ is lower semi-continuous on account of (2.7) and $u_{\alpha}^{(j)}$ is positive on $\bar{D}$. Accordingly, condition (M.3) is also satisfied. 
Therefore there exists an $m$-symmetric diffusion extension $X^{*}$ of $X$ from $\bar{D}$ to $\bar{D}^{*}$ admitting no killing on $F$. We can then use a general characterization theorem $[$ CF2, Theorem 7.7.3] to conclude that such an extension $X^{*}$ of $X$ is unique in law and its extended Dirichlet space $\left(\mathcal{F}_{e}^{*}, \mathcal{E}^{*}\right)$ is given by $(4.6)$ and $(4.7)$ as $\psi_{j}=\varphi_{j}, 1 \leq j \leq N$. In particular, (3.2) implies $1 \in \mathcal{F}_{e}^{*}, \mathcal{E}^{*}(1,1)=0$, so that $X^{*}$ is recurrent and consequently conservative. This also means the unique existence of an $m$-symmetric conservative diffusion extension $X^{*}$ of $X$ to $\bar{D}^{*}$.

TheOREM 4.2. $\operatorname{dim}\left(\mathcal{H}^{*}(D)\right)=N$ and

$$
\mathcal{H}^{*}(D)=\left\{\sum_{j=1}^{N} c_{j} \varphi_{j}: c_{j} \in \mathbb{R}\right\} .
$$

The m-symmetric conservative diffusion extension $X^{*}$ of the time changed $R B M X$ constructed in Proposition 4.1 is a reflecting extension of $X$ in the sense that the extended Dirichlet space $\left(\mathcal{F}_{e}^{*}, \mathcal{E}^{*}\right)$ of $X^{*}$ equals $(\mathrm{BL}(D), \mathbf{D} / 2)$ the reflected Dirichlet space of $X$.

Proof. $\quad$ By Proposition 4.1, $\left\{\varphi_{j} ; 1 \leq j \leq N\right\} \subset \mathcal{H}^{*}(D) \subset \mathrm{BL}(D)$. For $1 \leq j, k \leq$ $N$, let $c_{k}^{(j)}=c_{k}\left(\varphi_{j}\right)$. We claim that

$$
c_{k}^{(j)}=\delta_{j k}, \quad 1 \leq k \leq N .
$$

Let $\tau_{n}$ be the exit time of $Z$ from the set $\bar{D} \cap B_{n}(\mathbf{0}), n \geq 1$. Then $\left\{\varphi_{j}\left(Z_{\tau_{n}}\right)\right\}_{n \geq 1}$ is a bounded $\mathbf{Q}_{x}$-martingale and possesses an a.s. limit $\Phi$ with $\varphi_{j}(x)=\mathbf{E}^{Q_{x}}[\Phi]$. By (3.5),

$$
\Phi=\sum_{k=1}^{N} c_{k}^{(j)} \mathbf{1}_{\left\{Z_{\infty-}=\partial_{k}\right\}} .
$$

For $k \neq j$, put $F_{k, n}=C_{k} \cap\{|x|=n\}$. Then by (3.5) again

$$
\begin{aligned}
c_{k}^{(j)} \varphi_{k}(x) & =\lim _{n \rightarrow \infty} \mathbf{E}^{\mathbf{Q}_{x}}\left[\varphi_{j}\left(Z_{\tau_{n}}\right) \mathbf{1}_{\left\{Z_{\infty-}=\partial_{k}\right\}}\right] \leq \limsup _{n \rightarrow \infty} \mathbf{E}^{\mathbf{Q}_{x}}\left[\varphi_{j}\left(Z_{\tau_{n}}\right) \mathbf{1}_{\left\{Z_{\tau_{n}} \in C_{k}\right\}}\right] \\
& =\limsup _{n \rightarrow \infty} \mathbf{E}^{\mathbf{Q}_{x}}\left[\mathbf{Q}_{x}\left(Z_{\infty-} \circ \theta_{\tau_{n}}=\partial_{j}, Z_{\tau_{n}} \in C_{k} \mid \mathcal{F}_{\tau_{n}}\right)\right] \\
& \leq \lim _{n \rightarrow \infty} \mathbf{Q}_{x}\left(Z_{\infty-}=\partial_{j}, \sigma_{F_{k, n}}<\infty\right)=0,
\end{aligned}
$$

yielding $c_{k}^{(j)}=0, k \neq j$. Taking $\mathbf{Q}_{x^{-}}$expectation in (4.10) proves the claim (4.9).

Next for any $u \in \operatorname{BL}(D)$, let $u_{0}=u-\sum_{j=1}^{N} c_{j}(u) \varphi_{j}$. Then $u_{0} \in \operatorname{BL}(D)$ with $c_{j}\left(u_{0}\right)=0$ for every $1 \leq j \leq N$. So by Proposition 3.2, $u_{0} \in H_{e}^{1}(D)$. This establishes (4.8). The linear independence of $\left\{\varphi_{j} ; 1 \leq j \leq N\right\}$ follows from (4.9), while (4.6) and (4.8) yield the last assertion of the theorem.

REMARK 4.3. This theorem for the special domain (2.6) was stated in [CF2, Proposition 7.8.5]. We take this opportunity to mention that the proof of the latter given in the book [CF2] contained a flaw (on the third line of page 386), that should be corrected in the above way. 


\section{Partitions $\Pi$ of $F$ and all possible symmetric diffusion extensions $Y$ of a time changed RBM $X$.}

We continue to consider the $N$-points compactification $\bar{D}^{*}=\bar{D} \cup F$ of $\bar{D}$ introduced at the end of Section 1. A map $\Pi$ from the boundary set $F=\left\{\partial_{1}, \ldots, \partial_{N}\right\}$ onto a finite set $\widehat{F}=\left\{\widehat{\partial}_{1}, \ldots, \widehat{\partial}_{\ell}\right\}$ with $\ell \leq N$ is called a partition of $F$. We let $\bar{D}{ }^{\Pi, *}=\bar{D} \cup \widehat{F}$. We extend the map $\Pi$ from $F$ to $\bar{D}^{*}$ by setting $\Pi x=x, x \in \bar{D}$, and introduce the quotient topology on $\bar{D}^{\Pi, *}$ by $\Pi$. In other words, we employ $\mathcal{U}_{\Pi}=\left\{U \subset \bar{D}^{\Pi, *}\right.$ : $\Pi^{-1}(U)$ is an open subset of $\left.\bar{D}^{*}\right\}$ as the family of open subsets of $\bar{D}^{\Pi, *}$. Then $\bar{D}^{\Pi, *}$ is a compact Hausdorff space and may be called an $\ell$-points compactification of $\bar{D}$ obtained from $\bar{D}^{*}$ by identifying the points in the set $\Pi^{-1} \widehat{\partial}_{i} \subset F$ as a single point $\widehat{\partial}_{i}$ for each $1 \leq i \leq \ell$.

Given a partition $\Pi$ of $F$, the approaching probabilities $\widehat{\varphi}_{i}$ of the RBM $Z=\left(Z_{t}, \mathbf{Q}_{x}\right)$ to $\widehat{\partial}_{i} \in \widehat{F}$ are defined by

$$
\widehat{\varphi}_{i}(x)=\sum_{j \in \Pi^{-1} \widehat{\partial}_{i}} \varphi_{j}(x), \quad x \in \bar{D}, \quad 1 \leq i \leq \ell .
$$

As in the preceding section, we define the time changed process $X=\left(X_{t}, \zeta, \mathbf{P}_{x}\right)$ on $\bar{D}$ of $Z$ by means of a strictly positive bounded integrable function $f$ on $\bar{D}$. The measure $m(d x)=f(x) d x$ is extended from $\bar{D}$ to $\bar{D}^{\Pi \text {,* }}$ by setting $m(\widehat{F})=0$. Just as in Proposition 4.1, there exists then a unique $m$-symmetric conservative diffusion extension $X^{\Pi, *}$ of $X$ from $\bar{D}$ to $\bar{D}^{\Pi, *}$ and the Dirichlet form $\left(\mathcal{E}^{\Pi, *}, \mathcal{F}^{\Pi, *}\right)$ of $X^{\Pi, *}$ on $L^{2}\left(\bar{D}^{\Pi, *} ; m\right)\left(=L^{2}(D ; m)\right)$ admits the extended Dirichlet space $\left(\mathcal{F}_{e}^{\Pi, *}, \mathcal{E}^{\Pi, *}\right)$ expressed as

$$
\begin{gathered}
\mathcal{F}_{e}^{\Pi, *}=H_{e}^{1}(D) \oplus\left\{\sum_{i=1}^{\ell} c_{i} \widehat{\varphi}_{i}: c_{i} \in \mathbb{R}\right\} \subset \operatorname{BL}(D), \\
\mathcal{E}^{\Pi, *}(u, v)=\frac{1}{2} \mathbf{D}(u, v), \quad u, v \in \mathcal{F}_{e}^{\Pi, *} .
\end{gathered}
$$

$X^{\Pi, *}$ is recurrent. $\mathcal{E}^{\Pi, *}$ is a quasi-regular Dirichlet form on $L^{2}\left(\bar{D}^{\Pi, *} ; m\right)$.

We now prove that the family $\left\{X^{\Pi, *}: \Pi\right.$ is a partition of $\left.F\right\}$ exhausts all possible $m$-symmetric conservative diffusion extensions of the time changed RBM $X$ on $\bar{D}$.

Let $E$ be a Lusin space into which $\bar{D}$ is homeomorpically embedded as an open subset. The measure $m(d x)=f(x) d x$ on $\bar{D}$ is extended to $E$ by setting $m(E \backslash \bar{D})=0$. Let $Y=\left(Y_{t}, \mathbf{P}_{x}^{Y}\right)$ be an $m$-symmetric conservative diffusion process on $E$ whose part process on $\bar{D}$ is identical in law with $X$. We denote by $\left(\mathcal{E}^{Y}, \mathcal{F}^{Y}\right)$ and $\mathcal{F}_{e}^{Y}$ the Dirichlet form of $Y$ on $L^{2}(E ; m)$ and its extended Dirichlet space. We call $Y$ an $m$-symmetric conservative diffusion extension of $X$. The following theorem extends [CF1, Theorem 3.4]. See also [F4, Theorem 6.1] for analogous statements in a different context.

THEOREM 5.1. There exists a partition $\Pi$ of $F$ such that, as Dirichlet forms on $L^{2}(\bar{D} ; m)$,

$$
\left(\mathcal{E}^{Y}, \mathcal{F}^{Y}\right)=\left(\mathcal{E}^{\Pi, *}, \mathcal{F}^{\Pi, *}\right) .
$$


$Y$ under $\mathbf{P}_{g \cdot m}$ and $X^{\Pi \text {.* }}$ under $\mathbf{P}_{g \cdot m}^{\Pi, *}$ have the same finite dimensional distribution for any non-negative $g \in L^{2}(\bar{D} ; m)$. Furthermore, a quasi-homeomorphic image of $Y$ is identical with $X^{\Pi, *}$ in the sense of Theorem 8.2 in Appendix.

Proof. As has been noted in the preceding section, the extended Dirichlet space $\left(\mathcal{F}_{e}^{X}, \mathcal{E}^{X}\right)$ and the reflected Dirichlet space $\left(\left(\mathcal{F}^{X}\right)^{\text {ref }},\left(\mathcal{E}^{X}\right)^{\text {ref }}\right)$ of the Dirichlet form (4.4) are given by

$$
\begin{gathered}
\mathcal{F}_{e}^{X}=H_{e}^{1}(D), \quad \mathcal{E}^{X}=\frac{1}{2} \mathbf{D}, \\
\left(\mathcal{F}^{X}\right)^{\mathrm{ref}}=\mathrm{BL}(D)=H_{e}^{1}(D) \oplus \mathcal{H}^{*}(D), \quad\left(\mathcal{E}^{X}\right)^{\mathrm{ref}}=\frac{1}{2} \mathbf{D},
\end{gathered}
$$

respectively.

$\mathcal{E}^{Y}$ is a quasi-regular Dirichlet form on $L^{2}(E ; m)$ and $Y$ is properly associated with it by virtue of Ma and Röckner [MR]. By Chen-Ma-Röckner [CMR], $\mathcal{E}^{Y}$ is therefore quasi homeomorphic with a regular Dirichlet form. In particular, via a quasi homeomorphism $j$ in [CF2, Theorems 3.1.13]), we can assume that $E$ is a locally compact separable metric space, $\mathcal{E}^{Y}$ is a regular Dirichlet form on $L^{2}(E ; m), Y$ is an associated Hunt process on $E$, and $\widetilde{F}:=E \backslash \bar{D}$ is quasi-closed. Since $Y$ is a conservative extension of the non-conservative process $X, \widetilde{F}$ must be non $\mathcal{E}^{Y}$-polar. $Y$ can be also shown to be irreducible as in the proof of [CF2, Lemma 7.2.7 (ii)]. Thus we are in the same setting as in Section 7.1 of [CF2] and Theorem 7.1.6 in it applies to $Y$ and $\widetilde{F}$.

Every function in $\mathcal{F}_{e}^{Y}$ will be taken to be $\mathcal{E}^{Y}$-quasi continuous. As $Y$ is a diffusion with no killing inside, the jumping measure $J$ and the killing measure $k$ in the BeurlingDeny decomposition of $\mathcal{E}^{Y}$ vanish so that we have by [CF2, Theorem 7.1.6]

$$
\begin{gathered}
H_{e}^{1}(D) \subset \mathcal{F}_{e}^{Y} \subset \operatorname{BL}(D), \quad \mathcal{H}^{Y}:=\left\{\mathbf{H} u: u \in \mathcal{F}_{e}^{Y}\right\} \subset \mathcal{H}^{*}(D), \\
\mathcal{E}^{Y}(u, u)=\frac{1}{2} \mathbf{D}(u, u)+\frac{1}{2} \mu_{\langle\mathbf{H} u\rangle}^{c}(\widetilde{F}), \quad u \in \mathcal{F}_{e}^{Y}
\end{gathered}
$$

where $\mathbf{H} u(x)=\mathbf{E}_{x}^{Y}\left[u\left(Y_{\sigma_{\tilde{F}}}\right)\right], x \in E$.

Let us prove that

$$
\mu_{\langle u\rangle}^{c}(\widetilde{F})=0, \quad u \in \mathcal{H}^{Y} .
$$

To this end, we consider a finite measure $\nu$ on $E$ defined by

$$
\nu(B)=\int_{\bar{D}} \mathbf{P}_{x}^{Y}\left(Y_{\sigma_{\widetilde{F}}} \in B, \sigma_{\widetilde{F}}<\infty\right) m(d x), \quad B \in \mathcal{B}(E) .
$$

$\nu$ vanishes off $\widetilde{F}$ and charges no $\mathcal{E}^{Y}$-polar set. In view of [CF2, Lemma 5.2 .9 (i)], $\widetilde{F}$ is a quasi support of $\nu$ in the following sense: $\nu(E \backslash \widetilde{F})=0$ and $\widetilde{F} \subset \widehat{F}$ q.e. for any quasi closed set $\widehat{F}$ with $\nu(E \backslash \widehat{F})=0$.

Now, for $u \in \mathcal{H}^{Y},(4.8)$ and (5.7) imply that $u=\sum_{j=1}^{N} c_{j} \varphi_{j}$ for some constants $c_{j}$. Take $\widehat{F}=\left\{\xi \in E: u(\xi) \in\left\{c_{1}, \ldots, c_{N}\right\}\right\}$. Since $u$ is quasi continuous, $\widehat{F}$ is a quasi closed set. As $u$ is continuous along the sample path of $Y$ (cf. [CF2, Theorem 3.1.7]), we have 
$\nu(E \backslash \widehat{F})=\mathbf{P}_{m}\left(u\left(Y_{\sigma_{\widetilde{F}}}\right) \notin\left\{c_{1}, \ldots, c_{N}\right\}\right)=0$ on account of Proposition 3.2 and (4.9). Accordingly $\widetilde{F} \subset \widehat{F}$ q.e., namely, $u$ takes only finite values $\left\{c_{1}, \ldots, c_{N}\right\}$ q.e. on $\widetilde{F}$. By the energy image density property of $\mu_{\langle u\rangle}^{c}$ due to Bouleau and Hirsch [BH] (cf. [CF2, Theorem 4.3.8]), we thus get (5.9).

Relation (5.7) and Proposition 3.2(ii) imply that every function $u \in \mathcal{H}^{Y}(\subset \operatorname{BL}(D))$ admits a limit $u\left(\partial_{j}\right)$ at each boundary point $\partial_{j} \in F$ along the path of $Z$. Define an equivalence relation $\sim$ on $F$ by $\partial_{j} \sim \partial_{k}$ if and only if $u\left(\partial_{j}\right)=u\left(\partial_{k}\right)$ for every $u \in \mathcal{H}^{Y}$. Notice that, for every $1 \leq j \leq N$, there exists $u \in \mathcal{H}^{Y}$ with $u\left(\partial_{j}\right) \neq 0$. Otherwise, for the resolvent $\left\{G_{\alpha}^{Y}: \alpha>0\right\}$ of $Y, G_{\alpha}^{Y} 1 \in \mathcal{F}_{e}^{Y}(\subset \mathrm{BL}(D))$ approaches to zero at some $\partial_{j}$ along the path of $Z$, contradiction to the conservativeness of $Y$. Let $\Pi$ be the corresponding partition of $F$ : $\Pi$ maps $F$ onto $\left\{\widehat{\partial}_{1}, \ldots, \widehat{\partial}_{\ell}\right\}$ the set of all equivalence classes with respect to $\sim$. Then $\mathcal{H}^{Y}=\left\{\sum_{i=1}^{\ell} c_{i} \widehat{\varphi}_{i}: c_{i} \in \mathbb{R}\right\}$ for $\widehat{\varphi}_{i}$ defined by (5.1). Hence (5.2), (5.3), (5.7), (5.8) and (5.9) lead us to the desired identity (5.4).

Since the both Dirichlet forms share a common semigroup on $L^{2}(\bar{D} ; m)$, we get the first conclusion of the theorem. Further the Dirichlet spaces

$$
\left(E, m, \mathcal{E}^{Y}, \mathcal{F}^{Y}\right), \quad\left(\bar{D}^{\Pi, *}, m, \mathcal{E}^{\Pi, *}, \mathcal{F}^{\Pi, *}\right)
$$

are equivalent in the sense of Appendix (Section 8) by the identity map $\Phi$ from $\mathcal{F}_{b}^{Y}$ onto $\mathcal{F}_{b}^{\Pi, *}$ so that we get the second conclusion from Theorem 8.2.

REMARK 5.2. (i) For different choices of $f$, the family of all symmetric conservative extensions $Y$ of $X^{f}$ is invariant up to time changes because it shares a common family of extended Dirichlet spaces (5.2)-(5.3). The same can be said for more general time changed RBM $X^{\mu}$, which will be formulated in Section 7 .

(ii) We can replace the conservativeness assumption on $Y$ by a weaker one that $Y$ is a proper extension of $X$ with no killing on $E \backslash \bar{D}$. Then the above theorem remains valid if $X^{\Pi, *}$ is allowed to be replaced by its subprocess being killed upon hitting some (but not all) $\widehat{\partial}_{i}$.

REMARK 5.3 (Symmetric diffusion for a uniformly elliptic differential operator). Given measurable functions $a_{i j}(x), 1 \leq i, j \leq d$, on $D$ such that

$$
a_{i j}(x)=a_{j i}(x), \quad \Lambda^{-1}|\xi|^{2} \leq \sum_{1 \leq i, j \leq d} a_{i j}(x) \xi_{i} \xi_{j} \leq \Lambda|\xi|^{2}, \quad x \in D, \xi \in \mathbb{R}^{d}
$$

for some constant $\Lambda \geq 1$, we consider a Dirichlet form

$$
(\mathcal{E}, \mathcal{F})=\left(\boldsymbol{a}, H^{1}(D)\right)
$$

on $L^{2}(D)$ where

$$
\boldsymbol{a}(u, v)=\int_{D} \sum_{i, j=1}^{d} a_{i j}(x) \frac{\partial u}{\partial x_{i}}(x) \frac{\partial v}{\partial x_{j}}(x) d x, \quad u, v \in H^{1}(D) .
$$

If we replace the Dirichlet form $(2.2)$ on $L^{2}(D)$ and the associated RBM $Z$ on $\bar{D}$, re- 
spectively, by the Dirichlet form $(5.11)$ on $L^{2}(D)$ and the associated reflecting diffusion process on $\bar{D}$ constructed in [FTo], all results from Section 3 to Section 5 still hold without any change as we shall see now.

By this replacement, the extended Dirichlet space and the reflected Dirichlet space are still $H_{e}^{1}(D)$ and $\mathrm{BL}(D)$, respectively, although the inner product $\mathbf{D} / 2$ is replaced by $\boldsymbol{a}$. The transience of (5.11) is equivalent to that of (2.2). The space $\mathcal{H}^{*}(D)$ is now defined by (2.3) with $\boldsymbol{a}$ in place of $\mathbf{D} / 2$. But, by noting that $\boldsymbol{a}(c, c)=0$ for any constant $c$ and by taking the characterization of a Liouville domain stated below Definition 2.1 into account, we readily see that $D \in \mathcal{D}$ is a Liouville domain relative to (5.11) if and only if so it is relative to $(2.2)$.

REMARK 5.4 (All possible symmetric conservative diffusion extensions of a one-dimensional minimal diffusion). Consider a minimal diffusion $X$ on a one-dimensional open interval $I=\left(r_{1}, r_{2}\right)$ with no killing inside for which both boundaries $r_{1}, r_{2}$ are regular. Let $E$ be a Lusin space into which $I$ is homeomorphically embedded as an open subset. The speed measure $m$ of $X$ is extended to $E$ by setting $m(E \backslash I)=0$. Let $Y$ be an $m$-symmetric conservative diffusion extension of $X$ from $I$ to $E$. Then, by removing some $m$-polar open set for $Y$ from $\widetilde{F}=E \backslash I$, a homeomorphic image of $Y$ is identical with either the two point extension of $X$ to $\left[r_{1}, r_{2}\right]$ or its one-point extension to the one-point compactification of $I$. This fact was implicitly indicated in [F2, Section 5] and [F3, Section 5] without proof. This can be shown in a similar manner to the proof of Theorem 5.1 by establishing the counterpart of the identity (5.9) and by noting that, for the one-point and two-point extensions of $X$, every non-empty subset of the state space has a positive 1-capacity uniformly bounded away from zero due to the bound [CF2, (2.2.31)] and so a quasi-homeomorphism is reduced to a homeomorphism.

To put it another way, Theorem 5.1 reveals that the time changed RBM $X$ on an unbounded domain with $N$-Liouville branches has a very similar structure to the onedimensional diffusion only by changing two boundary points to $N$ boundary points.

We note that the connected sum of non-parabolic manifolds being studied by Kuz'menko and Molchanov [KM], Grigor'yan and Saloff-Coste [GS] bears a strong similarity to the present paper in the setting although the main concern in these papers was the heat kernel estimates.

\section{Characterization of $L^{2}$-generator of extension $Y$ by zero flux condi- tion at infnity.}

For a strictly positive bounded integrable function $f$ on $D$, we put $m(d x)=f(x) d x$ and denote by $(\cdot, \cdot)$ the inner product for $L^{2}(D ; m)$. Let $Y$ be any $m$-symmetric conservative diffusion extension of the time changed process $X=X^{f}=\left(X_{t}, \zeta, \mathbf{P}_{x}\right)$ of the RBM $Z$ on $\bar{D}$. Let $\Pi: F \mapsto\left\{\widehat{\partial}_{1}, \ldots, \widehat{\partial}_{\ell}\right\}, \ell \leq N$, be the corresponding partition of the boundary $F=\left\{\partial_{1}, \ldots, \partial_{N}\right\}$ appearing in Theorem 5.1. The Dirichlet form $\left(\mathcal{E}^{Y}, \mathcal{F}^{Y}\right)$ of $Y$ on $L^{2}(D ; m)$ is then described as 


$$
\left\{\begin{array}{l}
\mathcal{F}^{Y}=\left\{u=u_{0}+\sum_{i=1}^{\ell} c_{i} \widehat{\varphi}_{i}: u_{0} \in H_{e}^{1}(D) \cap L^{2}(D ; m), c_{i} \in \mathbb{R}\right\}, \\
\mathcal{E}^{Y}(u, v)=\frac{1}{2} \mathbf{D}(u, v), \quad u, v \in \mathcal{F}^{Y},
\end{array}\right.
$$

where $\widehat{\varphi}_{i}, 1 \leq i \leq \ell$, are defined by $(5.1)$.

Let $\mathcal{A}$ be the $L^{2}$-generator of $Y$, that is, $\mathcal{A}$ is a self-adjoint operator on $L^{2}(D ; m)$ such that $u \in \mathcal{D}(\mathcal{A}), \mathcal{A} u=v \in L^{2}(D ; m)$ if and only if $u \in \mathcal{F}^{Y}$ with $\mathcal{E}^{Y}(u, w)=-(v, w)$ for every $w \in \mathcal{F}^{Y}$. In view of Proposition 3.2, the condition (7.3.4) of [CF2] is fulfilled by $Y$. Therefore Theorem 7.7 .3 (vii) of $[\mathbf{C F 2}]$ is well applicable in getting the following characterization of $\mathcal{A}$ :

$$
u \in \mathcal{D}(\mathcal{A}) \quad \text { if and only if } \quad u \in \mathcal{D}(\mathcal{L}) \text { and } \mathcal{N}(u)\left(\widehat{\partial}_{i}\right)=0,1 \leq i \leq \ell .
$$

In this case, $\mathcal{A} u=\mathcal{L} u$.

Here $\mathcal{L}$ is a linear operator defined as follows: $u \in \mathcal{D}(\mathcal{L}), \mathcal{L} u=v \in L^{2}(D ; m)$ if and only if $u \in \operatorname{BL}(D) \cap L^{2}(D ; m)$ and $\mathbf{D} / 2(u, w)=-(v, w)$ for every $w \in H_{e}^{1}(D) \cap L^{2}(D ; m)$, or equivalently, for every $w \in C_{c}^{1}(\bar{D}) . \mathcal{N}(u)\left(\widehat{\partial}_{i}\right)$ is the flux of $u$ at $\widehat{\partial}_{i}$ defined by

$$
\mathcal{N}(u)\left(\widehat{\partial}_{i}\right)=\frac{1}{2} \mathbf{D}\left(u, \widehat{\varphi}_{i}\right)+\left(\mathcal{L} u, \widehat{\varphi}_{i}\right), \quad 1 \leq i \leq \ell .
$$

It can be readily verified that $u \in \mathcal{D}(\mathcal{L})$ if and only if $u \in \operatorname{BL}(D) \cap L^{2}(D ; m), \Delta u$ in the Schwartz distribution sense is in $L^{2}(D)$ and

$$
\mathbf{D}(u, w)+\int_{D} \Delta u(x) \cdot w(x) d x=0 \quad \text { for every } w \in C_{c}^{1}(\bar{D}) .
$$

In this case, $\mathcal{L} u(x)=1 / 2 f(x) \Delta u(x), x \in D$. The equation (6.1) can be interpreted as the requirement that the generalized normal derivative of $u$ vanishes on $\partial D$. Thus we have

TheOREM 6.1. $u \in \mathcal{D}(\mathcal{A})$ if and only if $u \in \mathrm{BL}(D) \cap L^{2}(D ; m), \Delta u$ in the Schwartz distribution sense belongs to $L^{2}(D)$, the equation (6.1) is satisfied and

$$
\left(\mathcal{N}(u)\left(\widehat{\partial}_{i}\right)=\right) \frac{1}{2} \mathbf{D}\left(u, \widehat{\varphi}_{i}\right)+\frac{1}{2} \int_{D} \Delta u(x) \widehat{\varphi}_{i}(x) d x=0, \quad 1 \leq i \leq \ell .
$$

In this case,

$$
\mathcal{A} u(x)=\frac{1}{2 f(x)} \Delta u(x), \quad \text { a.e. on } D .
$$

Suppose $u \in \mathcal{D}(\mathcal{A})$ is smooth on $\bar{D}$. Then $\partial u / \partial \boldsymbol{n}=0$ on $\partial D$ due to the condition (6.1) so that the zero flux condition (6.2) at $\widehat{\partial}_{j}$ can be expressed as

$$
\lim _{r \uparrow \infty} \int_{D \cap \partial B_{r}(\mathbf{0})} u_{r}(x) \widehat{\varphi}_{i}(x) d \sigma_{r}(d x)=0, \quad 1 \leq i \leq \ell,
$$

where $\sigma_{r}$ is the surface measure on $\partial B_{r}(\mathbf{0})$. 
The last part of Section $7.6\left(4^{\circ}\right)$ of $[$ CF2 $]$ has treated a very special case of the above where $D=\mathbb{R}^{d}, d \geq 3$, and $Y$ is the one-point reflection at the infinity of $\mathbb{R}^{d}$ of a time changed Brownian motion on $\mathbb{R}^{d}$.

In $[\mathbf{F 3}]$, the $L^{2}$-generator of any symmetric diffusion extension $Y$ of a onedimensional minimal diffusion $X$ is identified. In this case, the Dirichlet form of $Y$ admits its reproducing kernel which enables us to identify also the $C_{b}$-generator of $Y$, recovering the general boundary condition due to Feller and Itô-McKean.

\section{Extensions of more general time changed RBMs.}

All the results in Sections 4-6 except for (6.3) hold for more general time changed RBMs than $X^{f}$. Let $Z=\left(Z_{t}, \mathbf{Q}_{x}\right), f, X=X^{f}=\left(X_{t}, \zeta, \mathbf{P}_{x}\right), X^{*}=\left(X_{t}^{*}, \mathbf{P}_{x}^{*}\right)$ be as in Section 4.

We consider a positive finite measure $\mu$ on $\bar{D}$ charging no polar set with full quasisupport $\bar{D}$ relative to the Dirichlet form $(\mathcal{E}, \mathcal{F})$ of $(2.2)$. Let $A^{\mu}$ be the PCAF of $Z$ with Revuz measure $\mu$ and $X^{\mu}=\left(X_{t}^{\mu}, \zeta^{\mu}, \mathbf{P}_{x}^{\mu}\right)$ be the time changed process of $Z$ by $A^{\mu}$. The Markov process $X^{\mu}$ is $\mu$-symmetric and its Dirichlet form $\left(\mathcal{E}^{X^{\mu}}, \mathcal{F}^{X^{\mu}}\right)$ on $L^{2}(\bar{D} ; \mu)$ is given by

$$
\mathcal{E}^{X^{\mu}}=\frac{1}{2} \mathbf{D}, \quad \mathcal{F}^{X^{\mu}}=H_{e}^{1}(D) \cap L^{2}(\bar{D} ; \mu) .
$$

Proposition 7.1. It holds that

$$
\begin{gathered}
\mathbf{Q}_{x}\left(A_{\infty}^{\mu}<\infty\right)=1 \quad \text { for q.e. } x \in \bar{D} \\
\mathbf{P}_{x}^{\mu}\left(\zeta^{\mu}<\infty, X_{\zeta^{\mu}-}^{\mu}=\partial_{i}\right)=\varphi_{i}(x)>0
\end{gathered}
$$

Proof. Fix a strictly positive bounded integrable function $h_{0}$. By the transience of $Z$ and [CF2, Theorem A.2.13 (v)], $G_{0+}^{Z} h_{0}(x)<\infty$ for q.e. $x \in \bar{D}$. For integer $k \geq 1$, let

$$
\Lambda_{k}:=\left\{x \in \bar{D}: G_{0+}^{Z} h_{0}(x) \leq 2^{k}\right\} \quad \text { and } \quad h(x)=\sum_{k=1}^{\infty} 2^{-2 k} \mathbf{1}_{\Lambda_{k}}(x) h_{0}(x) .
$$

Then $h$ is a strictly positive bounded integrable function on $\bar{D}$ with $G_{0+}^{Z} h(x) \leq \mathbf{1}$ q.e. on $\bar{D}$. From $[\mathbf{C F 2},(4.1 .3)]$, we have

$$
\int_{\bar{D}} \mathbf{E}^{\mathbf{Q}_{x}}\left[A_{\infty}^{\mu}\right] h(x) d x=\left\langle G_{0+}^{Z} h, \mu\right\rangle \leq \mu(\bar{D})<\infty .
$$

It follows that $\mathbf{E}^{\mathbf{Q}_{x}}\left[A_{\infty}^{\mu}\right]<\infty$ a.e $x \in \bar{D}$ and hence q.e. $x \in \bar{D}$ by $[\mathbf{C F 2}$, Theorem A.2.13 (v)], yielding (7.2). (7.3) follows from (7.2) and Proposition 3.1.

Since $m(d x)=f(x) d x$ has its quasi-support $\bar{D}$ relative to $(\mathcal{E}, \mathcal{F})$, the Dirichlet form $\left(\mathcal{E}^{X}, \mathcal{F}^{X}\right)$ of (4.4) shares the common quasi-notion with $(\mathcal{E}, \mathcal{F})([\mathbf{C F 2}$, Theorem 5.2.11]). Hence the quasi-support of $\mu$ relative to $\left(\mathcal{E}^{X}, \mathcal{F}^{X}\right)$ is still $\bar{D}$.

The Dirichlet form $\left(\mathcal{E}^{*}, \mathcal{F}^{*}\right)$ on $L^{2}\left(\bar{D}^{*}, m\right)$ of $X^{*}$ is quasi-regular. According to the quasi-homeomorphism method already used in Section 4, we may assume it to be 
regular. The measure $\mu$ on $\bar{D}$ is extended to $\bar{D}^{*}$ by setting $\mu(F)=0$. We claim that the quasi-support of $\mu$ relative to this Dirichlet form equals $\bar{D}^{*}$ by using a criteria [CF2, Theorem 3.3.5].

Assume that $u \in \mathcal{F}^{*}$ is $\mathcal{E}^{*}$-quasi-continuous and that $u=0 \mu$-a.e. Then $\left.u\right|_{\bar{D}}$ is $\mathcal{E}^{X}$-quasi-continuous ([CF2, Theorem 3.3.8]) so that $u=0$ q.e. on $\bar{D}$. According to the same reference, there exists a Borel $m$-polar set $C \subset \bar{D}$ relative to $X^{*}$ such that $u(x)=0$ for every $x \in \bar{D} \backslash C$. Since $u$ is continuous along the path of $X^{*}$ ([CF2, Theorem 3.1.7]), we have for each $1 \leq i \leq N$

$$
\mathbf{P}_{m}^{*}\left(u\left(\partial_{i}\right)=\lim _{t \uparrow \sigma_{F}} u\left(X_{t}^{*}\right), \sigma_{C}=\infty, \sigma_{F}<\infty, X_{\sigma_{F}}^{*}=\partial_{i}\right)=\mathbf{P}_{m}\left(\zeta<\infty, X_{\zeta-}=\partial_{i}\right)>0,
$$

and so $u$ vanishes on $F$ and hence q.e. on $\bar{D}^{*}$, as was to be proved.

THEOREM 7.2. There exists a unique $\mu$-symmetric conservative diffusion $\widetilde{X}^{*, \mu}$ on $\bar{D}^{*}$ which is a q.e. extension of $X^{\mu}$ in the sense that the part of the former on $\bar{D}$ coincides in law with the latter for q.e. starting points $x \in \bar{D}$. The extended Dirichlet space of $\widetilde{X}^{*, \mu}$ equals $(\mathrm{BL}(D), \mathbf{D} / 2)$ the reflected Dirichlet space of $X^{\mu}$.

Proof. Let $B_{t}^{0}$ and $B_{t}$ be the PCAFs of $X$ and $X^{*}$, respectively, with Revuz measure $\mu$. According to [CF2, Proposition 4.1.10]

$$
B_{t}^{0}=B_{t \wedge \sigma_{F}} .
$$

Let $\widetilde{X}^{\mu}$ and $\widetilde{X}^{*, \mu}$ be the time changed processes of $X$ and $X^{*}$ by means of $B_{t}^{0}$ and $B_{t}$, respectively. The Markov process $\widetilde{X}^{\mu}$ is then the part of $\widetilde{X}^{*, \mu}$ on $\bar{D}$ by (7.5). Since $X^{*}$ is recurrent, so is $\widetilde{X}^{*, \mu}$ in view of $\left[\mathbf{C F 2}\right.$, Theorem 5.2.5]. Therefore $\widetilde{X}^{*, \mu}$ is a $\mu$-symmetric conservative diffusion extension of $\widetilde{X}^{\mu}$.

On the other hand, the Dirichlet form of $\widetilde{X}^{\mu}$ on $L^{2}(\bar{D} ; \mu)$ is identical with (7.1) the Dirichlet form of $X^{\mu}$ on $L^{2}(\bar{D} ; \mu)$, and consequently $\widetilde{X}^{*, \mu}$ is a q.e. extension of $X^{\mu}$. The last statement follows from the invariance of extended and reflected Dirichlet spaces under time changes by fully supported PCAFs.

The uniqueness of such a $\mu$-symmetric conservative Markovian extension of $X^{\mu}$ to $\bar{D}^{*}$ follows from [CF2, Theorem 7.7.3].

Similarly, all results in Section 4 and 5 with $\mu$ in place of $d m=f d x$ remain valid except for (6.3).

REMARK 7.3. One can give an alternative proof of Theorem 7.2 without invoking the time change of $X^{*}$ but still using the quasi-regularity of $\left(\mathcal{E}^{*}, \mathcal{F}^{*}\right)$. Indeed, the following proposition combined with (7.3) and [CF2, Theorem 7.7.3] readily yields Theorem 7.2 .

Each function in $\mathcal{F}_{e}^{*}$ is taken to be $\mathcal{E}^{*}$-quasi continuous. Define

$$
\widehat{\mathcal{F}}=\mathcal{F}_{e}^{*} \cap L^{2}(\bar{D} ; \mu) \quad \text { and } \quad \widehat{\mathcal{E}}(u, v)=\mathcal{E}^{*}(u, v)=\frac{1}{2} \mathbf{D}(u, v) \text { for } u, v \in \widehat{\mathcal{F}} .
$$


Proposition 7.4. (i) $(\widehat{\mathcal{E}}, \widehat{\mathcal{F}})$ is a quasi-regular Dirichlet form on $L^{2}\left(\bar{D}^{*} ; \mu\right)$.

(ii) Its associated strong Markov process $\widehat{X}$ on $\bar{D}^{*}$ is a $\mu$-symmetric conservative diffusion which is a q.e. extension of $X^{\mu}$.

(iii) Each $\partial_{j}$ is non- $\widehat{\mathcal{E}}$-polar.

Proof. (i) As $\bar{D}$ is a quasi-support of $\mu, u=0 \mu$-a.e. for $u \in \widehat{\mathcal{F}}$ implies $u=0$ a.e. on $\bar{D}$ and $\mathbf{D}(u, u)=0$. This together with the transience of $\left(\mathcal{F}_{e}^{*}, \mathcal{E}^{*}\right)$ implies that $(\widehat{\mathcal{E}}, \widehat{\mathcal{F}})$ is a well defined Dirichlet form on $L^{2}\left(\bar{D}^{*} ; \mu\right)$.

Since $\left(\mathcal{E}^{*}, \mathcal{F}^{*}\right)$ is a quasi-regular Dirichlet form on $L^{2}\left(\bar{D}^{*} ; m\right)$, by $[$ CF2, Remark 1.3.9], there is an increasing sequence of compact subsets $\left\{F_{k}\right\}$ of $\bar{D}^{*}$ so that

(a) there is an increasing sequence of compact subsets $\left\{F_{k}\right\}$ of $\bar{D}^{*}$ so that $\cup_{k \geq 1} \mathcal{F}_{F_{k}}^{*}$ is $\mathcal{E}_{1}^{*}$-dense in $\mathcal{F}^{*}$.

(b) there is an $\mathcal{E}_{1}^{*}$-dense of countable set $\Lambda_{0}:=\left\{f_{j} ; j \geq 1\right\}$ of bounded functions of $\mathcal{F}^{*}$ so that $\left\{f_{j} ; j \geq 1\right\} \subset C\left(\left\{F_{k}\right\}\right)$ and they separate points of $\cup_{k \geq 1} F_{k}$.

By the contraction of the Dirichlet form, we may and do assume without loss of generality that for every integer $n \geq 1$ and $f \in \Lambda_{0},((-n) \vee f) \wedge n \in \Lambda_{0}$. We claim that $\cup_{k \geq 1} \mathcal{F}_{F_{k}, b}^{*} \subset$ $\cup_{k \geq 1} \widehat{\mathcal{F}}_{F_{k}, b}$ is $\widehat{\mathcal{E}}_{1}$-dense in $\widehat{\mathcal{F}}_{b}$. Let $u \in \widehat{\mathcal{F}}_{b}$. Since $\widehat{\mathcal{F}}_{b}=\mathcal{F}_{b}^{*}$, there are $u_{k} \in \mathcal{F}_{F_{k}}^{*}$ so that $u_{k} \rightarrow$ $u$ in $\mathcal{E}_{1}^{*}$-norm. Using truncation if needed, we may and do assume $\left\|u_{k}\right\|_{\infty} \leq\|u\|_{\infty}+1$. Taking a subsequence if needed, we may also assume that $u_{k}$ converges to $u \mathcal{E}^{*}$-q.e. on $\bar{D}^{*}$. Since $\mu$ is a finite smooth measure, we conclude that $u_{k}$ is $\widehat{\mathcal{E}}_{1}$-convergent to $u$. This proves the claim. As $\widehat{\mathcal{F}}_{b}$ is $\widehat{\mathcal{E}}_{1}$ dense in $\widehat{\mathcal{F}}$, it follows that $\left\{F_{k}\right\}$ is an $\widehat{\mathcal{E}}$-nest on $\bar{D}^{*}$.

A similar argument shows that $\Lambda_{0} \subset \widehat{\mathcal{F}}_{b}=\mathcal{F}_{b}^{*}$ is $\widehat{\mathcal{E}}_{1}$-dense in $\widehat{\mathcal{F}}_{b}$ and hence in $\widehat{\mathcal{F}}$. This proves the assertion (i).

(ii) Since $1 \in \widehat{\mathcal{F}}$ and $\mathbf{D}(1,1)=0$, the associated $\mu$-symmetric diffusion $\widehat{X}$ on $\bar{D}^{*}$ is recurrent and conservative. For $R>r$, take $\psi \in C_{c}^{\infty}(\bar{D})$ with $\psi=1$ on $B_{R+1}(\mathbf{0})$. Then, for any bounded $u \in \widehat{\mathcal{F}}, \psi u \in H_{e}^{1}(D)$ and so

$\left\{v \in \widehat{\mathcal{F}}: v=0\right.$ q.e. on $\left.\bar{D}^{*} \backslash B_{R}(\mathbf{0})\right\}=\left\{v \in H_{e}^{1}(D) \cap L^{2}(\bar{D} ; \mu): v=0\right.$ q.e. on $\left.\bar{D} \backslash B_{R}(\mathbf{0})\right\}$,

namely, the part of $\widehat{\mathcal{E}}$ on $\bar{D} \cap B_{R}(\mathbf{0})$ coincides with the part of $\mathcal{E}^{X^{\mu}}$ on $\bar{D} \cap B_{R}(\mathbf{0})$. By letting $R \rightarrow \infty$, we see that the part of $\widehat{\mathcal{E}}$ on $\bar{D}$ coincides with $\mathcal{E}^{X^{\mu}}$, proving (ii).

(iii) The non- $\widehat{\mathcal{E}}$-polarity of $\partial_{j}$ follows from (ii) and (7.3).

\section{Appendix: equivalence and quasi-homeomorphism.}

In dealing with boundary problems for symmetric Markov processes, it is convenient to introduce an equivalence of Dirichlet spaces following [FOT, A.4] as will be stated below.

We say that a quadruplet $(E, m, \mathcal{E}, \mathcal{F})$ is a Dirichlet space if $E$ is a Hausdorff topological space with a countable base, $m$ is a $\sigma$-finite positive Borel measure on $E$ and $\mathcal{E}$ with domain $\mathcal{F}$ is a Dirichlet form on $L^{2}(E ; m)$. The inner product in $L^{2}(E ; m)$ is denoted by $(\cdot, \cdot)_{E}$. For a given Dirichlet space $(E, m, \mathcal{E}, \mathcal{F})$, the notions of an $\mathcal{E}$-nest, an 
$\mathcal{E}$-polar set, an $\mathcal{E}$-quasi-continuous numerical function and ' $\mathcal{E}$-quasi-everywhere' (' $\mathcal{E}$-q.e.' in abbreviation) are defined as in [CF2, Definition 1.2.12]. The quasi-regularity of the Dirichlet space is defined just as in [CF2, Definition 1.3.8]. We note that the space $\mathcal{F}_{b}=\mathcal{F} \cap L^{\infty}(E ; m)$ is an algebra.

REMARK 8.1. In Section 1.2 and the first half of Section 1.3 of [CF2], it is assumed that

$$
\operatorname{supp}[m]=E .
$$

We need not assume it. Generally, if we let $E^{\prime}=\operatorname{supp}[m]$, then $E \backslash E^{\prime}$ is $\mathcal{E}$-polar according to the definition of the $\mathcal{E}$-polarity. If $(E, m, \mathcal{E}, \mathcal{F})$ is quasi-regular, so is $\left(E^{\prime},\left.m\right|_{E^{\prime}}, \mathcal{E}, \mathcal{F}\right)$ accordingly. Therefore we may assume (8.1) if we like by replacing $E$ with $E^{\prime}$.

Given two Dirichlet spaces

$$
(E, m, \mathcal{E}, \mathcal{F}), \quad(\tilde{E}, \tilde{m}, \tilde{\mathcal{E}}, \tilde{\mathcal{F}}),
$$

we call them equivalent if there is an algebraic isomorphism $\Phi$ from $\mathcal{F}_{b}$ onto $\tilde{\mathcal{F}}_{b}$ preserving three kinds of metrics: for $u \in \mathcal{F}_{b}$

$$
\|u\|_{\infty}=\|\Phi u\|_{\infty}, \quad(u, u)_{E}=(\Phi u, \Phi u)_{\tilde{E}}, \mathcal{E}(u, u)=\tilde{\mathcal{E}}(\Phi u, \Phi u)
$$

One of the two equivalent Dirichlet spaces is called a representation of the other.

The underlying spaces $E, \tilde{E}$ of two Dirichlet spaces (8.2) are said to be quasihomeomorphic if there exist $\mathcal{E}$-nest $\left\{F_{n}\right\}, \tilde{\mathcal{E}}$-nest $\left\{\tilde{F}_{n}\right\}$ and a one to one mapping $q$ from $E_{0}=\cup_{n=1}^{\infty} F_{n}$ onto $\tilde{E}_{0}=\cup_{n=1}^{\infty} \tilde{F}_{n}$ such that the restriction of $q$ to each $F_{n}$ is a homeomorphism onto $\tilde{F}_{n} .\left\{F_{n}\right\},\left\{\tilde{F}_{n}\right\}$ are called the nests attached to the quasihomemorphism q. Any quasi-homeomorphism is quasi-notion-preseving.

We say that the equivalnce $\Phi$ of two Dirichlet spaces (8.2) is induced by a quasihomeomorphism $q$ of the underlying spaces if

$$
\Phi u(\tilde{x})=u\left(q^{-1}(\tilde{x})\right), \quad u \in \mathcal{F}_{b}, \quad \tilde{m} \text {-a.e. } \tilde{x} .
$$

Then $\tilde{m}$ is the image measure of $m$ and $(\tilde{\mathcal{E}}, \tilde{\mathcal{F}})$ is the image Dirichlet form of $(\mathcal{E}, \mathcal{F})$.

TheOREM 8.2. Assume that two Dirichlet spaces (8.2) are quasi-regular and that they are equivalent. Let $X=\left(X_{t}, \mathbb{P}_{x}\right)$ (resp. $\left.\tilde{X}=\left(\tilde{X}_{t}, \tilde{\mathbb{P}}_{x}\right)\right)$ be an m-symmetric right process on $E$ (resp. an $\tilde{m}$-symmetric right process on $\tilde{E})$ properly associated with $(\mathcal{E}, \mathcal{F})$ on $L^{2}(E ; m)\left(\operatorname{resp} .(\tilde{\mathcal{E}}, \tilde{\mathcal{F}})\right.$ on $\left.L^{2}(\tilde{E} ; \tilde{m})\right)$. Then the equivalence is induced by a quasihomeomorphism $q$ with attached nests $\left\{F_{n}\right\},\left\{\tilde{F}_{n}\right\}$ such that $\tilde{X}$ is the image of $X$ by $q$ in the following sense: there exist an $m$-inessential Borel subset $N$ of E containing $\cap_{n=1}^{\infty} F_{n}^{c}$ and an $\tilde{m}$-inessential Borel subset $\tilde{N}$ of $\tilde{E}$ containing $\cap_{n=1}^{\infty} \tilde{F}_{n}^{c}$ so that $q$ is one to one from $E \backslash N$ onto $\tilde{E} \backslash \tilde{N}$ and

$$
\tilde{X}_{t}=q\left(X_{t}\right), \quad \tilde{\mathbb{P}}_{\tilde{x}}=\mathbb{P}_{q^{-1} \tilde{x}}, \quad \tilde{x} \in \tilde{E} \backslash \tilde{N} .
$$

Proof. Since both Dirichlet spaces in (8.2) are assumed to be quasi-regular, they 
are equivalent to some regular Dirichlet spaces and the equivalences are induced by some quasi-homeomorphisms $q_{1}, q_{2}$ in view of [CF2, Theorem 1.4.3]. Since two Dirichlet spaces in (8.2) are also assumed to be equivalent, so are the corresponding two regular Dirichlet spaces, the equivalence being induced by a quasi-homeomorphism $q_{3}$ on account of [FOT, Theorem A.4.2] combined with [CF2, Theorem 1.2.14]. Hence the equivalence of the quasi-regular Dirichlet spaces in (8.2) is induced by the quasi-homeomorphism $q=q_{1} \circ q_{3} \circ q_{2}^{-1}$ between $E$ and $\tilde{E}$. Let $\left\{F_{n}\right\},\left\{\tilde{F}_{n}\right\}$ be the nests attached to $q$.

According to [CF2, Theorem 3.1.13], we may assume without loss of generality that both $X$ and $\tilde{X}$ are Borel right processes. Further the $\mathcal{E}$-polarity is equivalent to the $m$-polar for $X$. By virtue of [CF2, Theorem A.2.15], we can therefore find an $m$ inessential Borel set $N_{1} \subset E$ containing $\cap_{n=1}^{\infty} F_{n}^{c}$. Consider the set $\tilde{N}_{1} \subset \tilde{E}$ defined by $q\left(E \backslash N_{1}\right)=\tilde{E} \backslash \tilde{N}_{1} . \tilde{N}_{1}$ is an $\tilde{\mathcal{E}}$-polar Borel set and $q$ is one to one from $E \backslash N_{1}$ onto $\tilde{E} \backslash \tilde{N}_{1}$.

Define the process $\widehat{X}=\left(\widehat{X}_{t}, \widehat{\mathbb{P}}_{\tilde{x}}\right)_{\tilde{x} \in \tilde{E} \backslash \tilde{N}_{1}}$ by

$$
\widehat{X}_{t}=q\left(X_{t}\right), \quad \widehat{\mathbb{P}}_{\tilde{x}}=\mathbb{P}_{q^{-1} \tilde{x}}, \quad \tilde{x} \in \tilde{E} \backslash \tilde{N}_{1}
$$

On account of [FFY, Lemma 3.1], we can then see that $\widehat{X}$ is an $\tilde{m}$-symmetric Markov process on $\tilde{E} \backslash \tilde{N}_{1}$ properly associated with the Dirichlet form $(\tilde{\mathcal{E}}, \tilde{\mathcal{F}})$ on $L^{2}(\tilde{E} ; \tilde{m})$. Since the $\tilde{m}$-symmetric Borel right process $\tilde{X}$ is also properly associated with the Dirichlet form $(\tilde{\mathcal{E}}, \tilde{\mathcal{F}})$ on $L^{2}(\tilde{E} ; \tilde{m})$, the same method as in the proof of [CF2, Theorem 3.1.12] combined with [CF2, Theorem A.2.15] leads us to finding an $\tilde{m}$-inessential Borel set $\tilde{N}$ containing $\tilde{N}_{1}$ for $\tilde{X}$ such that the Markov processes $\left.\tilde{X}\right|_{\tilde{E} \backslash \tilde{N}}$ and $\left.\widehat{X}\right|_{\tilde{E} \backslash \tilde{N}}$ are identical in law. It now suffices to define the set $N$ by $E \backslash N=q^{-1}(\tilde{E} \backslash \tilde{N})$.

REMARK 8.3. Owing to the works of Albeverio, Ma, Röckner and Fitzsimmons, the quasi-regularity of a Dirichlet form has been known to be not only a sufficient condition but also a necessary one for the existence of a properly associated right process. It is further shown in $[\mathbf{C M R}]$ that a Dirichlet form is quasi-regular if and only if it is quasihomeomorphic to a regular Dirichlet form on a locally compact separable metric space. These facts are formulated by Theorem 1.5.3 and Theorem 1.4.3, respectively, of [CF2] under the assumption (8.1) which is not needed actually. But we may assume it without loss of generality as will be seen below.

Indeed, let $E$ be a Lusin space, $m$ be a $\sigma$-finite measure on $E$ and $X$ be an $m$ symmetric Borel right process on $E$. Then, for $E_{0}=\operatorname{supp}[m], E \backslash E_{0}$ is an $m$-negligible open set so that it is $m$-polar for $X$ by [CF2, Theorem A.2.13 (iii)]. Hence, by [CF2, Theorem A.2.15], there exists a Borel set $E_{1} \subset E_{0}$ such that $E \backslash E_{1}$ is $m$-inessential for $X . E_{1}$ is the support of $\left.m\right|_{E_{1}}$ because, for any $x \in E_{1}$ and any neighborhood $O(x)$ of $x$, $m\left(O(x) \cap E_{1}\right)=m(O(x))-m\left(O(x) \cap\left(E \backslash E_{1}\right)\right)>0$. Hence it suffices to replace $E$ by $E_{1}$.

In Theorem 5.1, the extension process $Y$ is assumed to live on a Lusin space $E$ into which $\bar{D}$ is homeomorphically embedded as an open subset. In this particular case, the above set $E_{1}$ can be choosen to contain $\bar{D}$ on account of the proof of [CF2, Theorem A.2.15]. Therefore, in Theorem 5.1 (resp. Remark 5.4), we can assume more strongly that $\bar{D}$ (resp. $I$ ) is homeomorphically embedded into the state space $E$ of $Y$ as a dense open subset. 


\section{References}

[BH] N. Bouleau and F. Hirsch, Dirichlet Forms and Analysis on Wiener Space, De Gruyter, 1991.

[B] M. Brelot, Etude et extension du principe de Dirichlet, Ann. Inst. Fourier, 5 (1953/54), 371419 .

[C] Z.-Q. Chen, On reflecting diffusion processes and Skorokhod decompositions, Probab. Theory Relat. Fields, 94 (1993), 281-315.

[CF1] Z.-Q. Chen and M. Fukushima, On unique extension of time changed reflecting Brownian motions, Ann. Inst. Henri Poincaré Probab. Statist., 45 (2009), 864-875.

[CF2] Z.-Q. Chen and M. Fukushima, Symmetric Markov Processes, Time Change and Boundary Theory, Princeton University Press, 2011.

[CF3] Z.-Q. Chen and M. Fukushima, One-point reflections, Stochastic Process Appl., 125 (2015), 1368-1393.

[CMR] Z.-Q. Chen, Z.-M. Ma and M. Röckner, Quasi-homeomorphisms of Dirichlet forms, Nagoya Math. J., 136 (1994), 1-15.

[DL] J. Deny and J. L. Lions, Les espaces du type de Beppo Levi, Ann. Inst. Fourier, 5 (1953/54), 305-370.

[FFY] X. Fang, M. Fukushima and J. Ying, On regular Dirichlet subspaces of $H^{1}(I)$ and associated linear diffusions, Osaka J. Math., 42 (2005), 27-41.

[F1] M. Fukushima, On boundary conditions for multi-dimensional Brownian motions with symmetric resolvent densities, J. Math. Soc. Japan, 21 (1969), 58-93.

[F2] M. Fukushima, From one dimensional diffusions to symmetric Markov processes, Stochastic Process Appl., 120 (2010), 590-604.

[F3] M. Fukushima, On general boundary conditions for one-dimensional diffusions with symmetry, J. Math. Soc. Japan, 66 (2014), 289-316.

[F4] M. Fukushima, Liouville property of harmonic functions of finite energy for Dirichlet forms, to appear in Stochastic Partial Differential Equations and Related Fields, Springer Proc. in Math. and Stat.

[FOT] M. Fukushima, Y. Oshima and M. Takeda, Dirichlet Forms and Symmetric Markov Processes, De Grruyter, 1994, 2nd Edition, 2010.

[FTa] M. Fukushima and M. Takeda, Markov Processes (in Japanese), Baifukan, Tokyo, 2008, Chinese translation by P. He, (ed. J. Ying), Science Press, Beijing, 2011.

[FTo] M. Fukushima and M. Tomisaki, Construction and decomposition of reflecting diffusions on Lipschitz domains with Hölder cusps, Probab. Theory Relat. Fields, 106 (1996), 521-557.

[GS] A. Grigor'yan and L. Saloff-Coste, Heat kernels on manifolds with ends, Ann. Inst. Fourier, Grenoble 59 (2009), 1917-1997.

[HK] D. A. Herron and P. Koskella, Uniform, Sobolev extension and quasiconformal circle domains, J. Anal. Math., 57 (1991), 172-202.

[ J ] P. W. Jones, Quasiconformal mappings and extendibility of functions in Sobolev spaces, Acta Math., 147 (1981), 71-88.

[KM] Y. Kuz'menko and S. Molchanov, Counterexamples to Liouville-type theorems, Moscow Univ. Math. Bull., 34 (1979), 35-39.

[MR] Z.-M. Ma and M. Röckner, Introduction to the Theory of (non-symmetric) Dirichlet Forms, Springer, 1992.

[M] V. G. Maz’ja, Sobolev Spaces, Springer, 1985.

[P] R. G. Pinsky, Transience/recurrence for normally reflected Brownian motion in unbounded domains, Ann. Probab., 37 (2009), 676-686.

\author{
Zhen-Qing CHEN \\ Department of Mathematics \\ University of Washington \\ Seattle \\ WA 98195, USA \\ E-mail: zqchen@uw.edu
}

\author{
Masatoshi Fukushima \\ Branch of Mathematical Science \\ Osaka University \\ Toyonaka \\ Osaka 560-8531, Japan \\ E-mail: fuku2@mx5.canvas.ne.jp
}

